\title{
Transient Heat Analysis in a Spherical Domain of Combustible Material
}

\author{
Ramoshweu Solomon Lebelo \\ Department of Mathematics,Vaal University of Technology, Private Bag X021, Vanderbijlpark 1911, South Africa
}

\begin{abstract}
Analysis of transient heat in a stockpile of combustible material modelled in a spherical domain is considered in this article. The sphere loses heat by convection to the atmosphere due to exothermic chemical reaction taking place in the reactive stockpile. The complicated chemical reaction taking place in this problem is tackled by assuming a one-step finite rate irreversible Arrhenius kinetics. The differential equation governing the problem is solved numerically by using the FDM (finite difference method) that is very essential for spatial derivatives discretization. The transient heat is analyzed for various thermo-physical parameters embedded in the system and the results are discussed accordingly.
\end{abstract}

Key words: Transient heat, reactive sphere, FDM, exothermic chemical reaction.

\section{Introduction}

Spontaneous self-heating in as stockpile of reactive material is due to exothermic chemical reaction where oxygen reacts with carbon containing material in which carbon dioxide is emitted. The emission of carbon dioxide is of environmental importance and previous studies showed that about $80 \%$ of carbon dioxide emitted due to self-heating contributes to Greenhouse effect [1-3]. Generally all municipal dumping sites have stockpiles of combustible materials and if these are not well managed, self-heating may take place to give fires that are hazardous to living species, environment and industry $[4,5]$. Most of reactive stockpiles have materials such as wool, cotton, hay, industrial wastes and other carbon containing substances. Self-heating due to exothermic chemical reaction is a complicated process that involves many radicals [6, 7]. The complicated chemistry taking place is tackled using a one-step decomposition kinetics [8, 9]. The mathematical modelling provides necessary understanding into this phenomenon, and due to nonlinear interactions or reacting species, it is practically not possible to obtain

Corresponding author: Ramoshweu Solomon Lebelo, Ph.D., senior lecturer, research fields: fluid dynamics, heat transfer. exact solution to the differential equation governing the problem $[10,11]$.

Analysis of transient heat in a reactive slab was investigated in Ref. [12], and in Ref. [13] the analysis was studied in a long cylindrical pipe. The latter applied regular perturbation technique coupled with computer-extended series and a special type of Hermite-Pade' approximation to solve the governing equation numerically. This article considers transient heat analysis in a spherical domain of combustible material. The governing equation is solved by using FDM (finite difference method). The mathematical formulation in this work is done in Section 2 and the numerical method applied is presented in Section 3. Section 4 gives detailed discussion of results and graphical solutions to the problem due to various thermo-physical parameters embedded within the system.

\section{Mathematical Formulation}

A stockpile of combustible material modelled in a spherical domain with constant thermal conductivity $k$, is considered in this study. The stockpile is assumed to be subjected to a one-step Arrhenius kinetics with the possibility of heat loss to the ambient. Fig. 1 depicts 


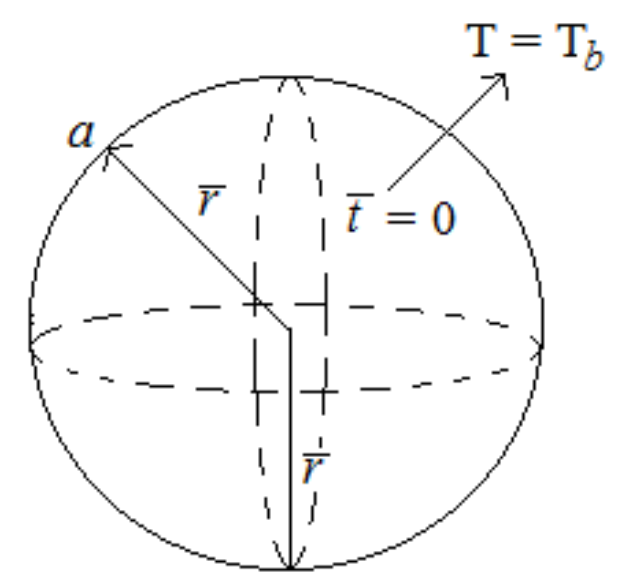

Fig. 1 Geometry of the problem.

the geometry of the problem.

Neglecting the reactant consumption, the one-dimensional governing equation where the sphere of radius $\bar{r}=a$ with temperature, initially at $T=T_{0}$, is given by Refs. [11, 12]

$$
p c_{p} \frac{\partial T}{\partial \bar{t}}=k \frac{1}{\bar{r}^{2}} \frac{\partial}{\partial r}\left(\bar{r}^{2} \frac{\partial T}{\partial r}\right)+Q A C\left(\frac{K T}{v l}\right)^{m} e-E / R T-\varphi\left(T-T_{b}\right)
$$

The initial condition is

$$
T(\bar{r}, 0)=T_{0}
$$

with boundary conditions

$$
\frac{\partial T}{\partial \bar{r}}(0, \bar{t})=0 ; T(a, \bar{t})=T_{b}
$$

Here $T$ is the sphere's absolute temperature, $T_{0}$ the initial temperature of the sphere and $T_{b}$ the ambient temperature. $p$ is the density, $c_{p}$ the specific heat at constant pressure, $k$ the thermal conductivity of the material, $Q$ the heat of reaction, $A$ the rate constant and $C$ the reactant concentration. We also have $K$ as the Boltzmann's constant, $v$ the vibration frequency, $l$ the Planck's number, $E$ the activation energy and $R$ the universal gas number. $m$ represents the numerical exponent and takes the following values, -2 for sensitized, 0 for Arrhenius and 0.5 for bimolecular kinetics and $\varphi$ is the heat loss parameter $[1-3,11$, 12].

The following dimensionless parameters are introduced to Eqs. (1)-(3).

$$
\begin{gathered}
\theta=\frac{E\left(T-T_{b}\right)}{R T_{b}^{2}}, \theta=\frac{E\left(T_{0}-T_{b}\right)}{R T_{b}^{2}}, r=\frac{\bar{r}}{a}, \mu=\frac{R T_{b}}{E}, \\
t=\frac{k \bar{t}}{p c_{p} a^{2}}, a=\frac{a \varphi}{k}, \\
\lambda=\left(\frac{K T_{b}}{v l}\right)^{m} \frac{Q A E a^{2} C}{k R T_{b}^{2}} \exp \left(-\frac{E}{R T_{b}}\right)
\end{gathered}
$$

Eqs. (1)-(3) take the following forms:

$$
\frac{\partial \theta}{\partial t}=\frac{1}{r^{2}} \frac{\partial}{\partial r}\left(r^{2} \frac{\partial \theta}{\partial r}\right)+\lambda(1+\mu \theta)^{m} e^{\theta /(1+\mu \theta)}-\alpha \theta
$$

The initial condition is

$$
\theta(r, 0)=\theta_{0},
$$

and boundary conditions are

$$
\frac{\partial \theta}{\partial r}(0, t)=0 ; \theta(1, t)=0,
$$

where $\theta$ is the dimensionless temperature, $\theta_{0}$ is the dimensionless initial temperature, $\lambda$ is the Frank-Kamenetskii parameter, also called the rate of reaction parameter, $\mu$ is the activation energy parameter, $r$ is the dimensionless radial distance and $\alpha$ is the heat loss parameter.

\section{Numerical Approach}

The partial differential Eq. (5), including its initial and boundary conditions, is solved using the FDM algorithm. This method uses a mesh of nodes and the time coordinate is introduced in addition to spatial mesh. The governing equation is approximated by the scheme and the resulting system of algebraic equations is solved at each time level so that the approximated values of the solution are obtained at the spatial nodes. The explicit scheme is used in this case, where space domain is subdivided into equal size of subintervals to give a mesh of points. A mesh of points in time is also created. The forward difference 
approximation is applied for the time derivative and the centered difference formula is used for the spatial derivatives.

The second order accurate difference formula for Eq. (5) is thus:

$\frac{\theta_{i}^{j+1}-\theta_{i}^{j}}{\Delta t}=\frac{1}{r^{2} \Delta r^{2}}\left(r_{i+\frac{1}{2}}^{2} \theta_{i+1}^{j}-2 r_{i} \theta_{i}^{j}+r_{i-\frac{1}{2}}^{2} \theta_{i-1}^{j}\right)+\lambda\left(1+\mu \theta_{i}^{j}\right)^{m} e^{\theta_{i}^{j} /\left(1+\mu \theta_{i}^{j}\right)}-\alpha \theta_{i}^{j}$,

where $r_{i} ; i=1,2, \ldots N$ are called nodal positions along the radial direction, ${ }_{i \pm \frac{1}{2}}$ are positions of points located half-way between $r_{i}$ and $r_{i+1}, \Delta r$ is the mesh spacing, and $j=1,2, \ldots, M$ is the index of time.

Another second order finite difference formula at the center of the sphere is obtained by using fictitious node located at $r=-\Delta r$. Therefore the boundary condition at the center of the sphere, using centered differences, is thus:

$$
\frac{\theta_{2}^{j}-\theta_{-2}^{j}}{2 \Delta r}=0
$$

and the last grid point is

$$
\theta_{N}=0
$$

The initial condition is expressed as

$$
\theta_{i}(0)=\theta_{0}
$$

The FDM algorithm applied to solve Eqs. (8)-(11) is built in most of mathematical software, and in this case the equations were solved using Maple.

\section{Results and Discussion}

It is assumed that at the initial stage, $t=0$, the temperature of the sphere is equal to that of the surrounding. But at $t>0$, the temperature of the sphere exceeds that of the ambient due to self-heating caused by the trapped oxygen reacting with the reactive material within the sphere. Figs. 2-5 illustrate the behavior of the temperature in response to variation of thermo-physical parameters embedded in the system. Figs. 2 and 3 illustrate the behavior of temperature as $t$ increases while other thermo-physical parameters, $\lambda, \mu, m$ and $\alpha$, are kept constant. The general trend is that the temperature of the sphere is highest along the center and lowest at its surface as heat loss to the ambient takes place for $t>0$. We observe also from Figs. 2 and 3 that the sphere's temperature attains its steady state value, and once the steady state value is reached, no further increase in temperature is experienced. In Fig. 4 we observe how temperature varies as $\lambda$, the rate of reaction parameter, is increased. An increase in $\lambda$ shows a corresponding increase in temperature fields of the sphere due to exothermic chemical reaction taking place within the system. As a result, we see high values of temperature attained, and if $\lambda$ keeps on increasing without appropriate

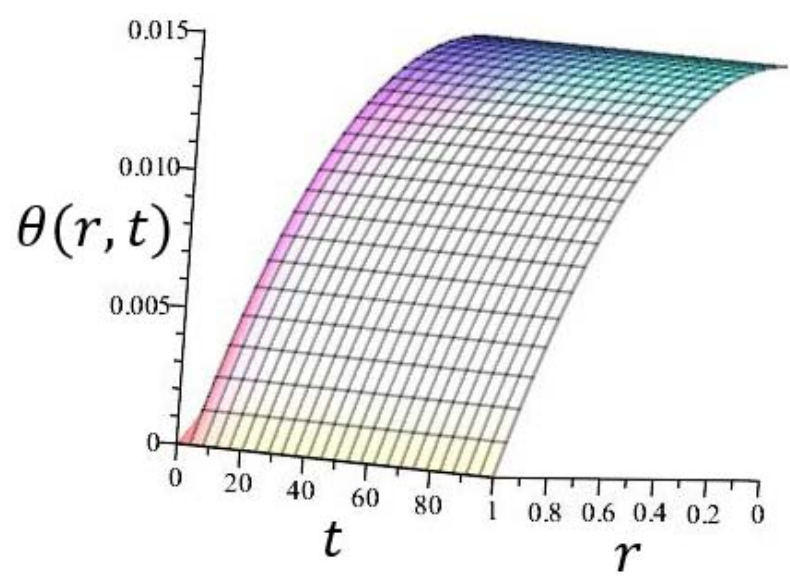

Fig. 2 Temperature variation with time, 3-D.

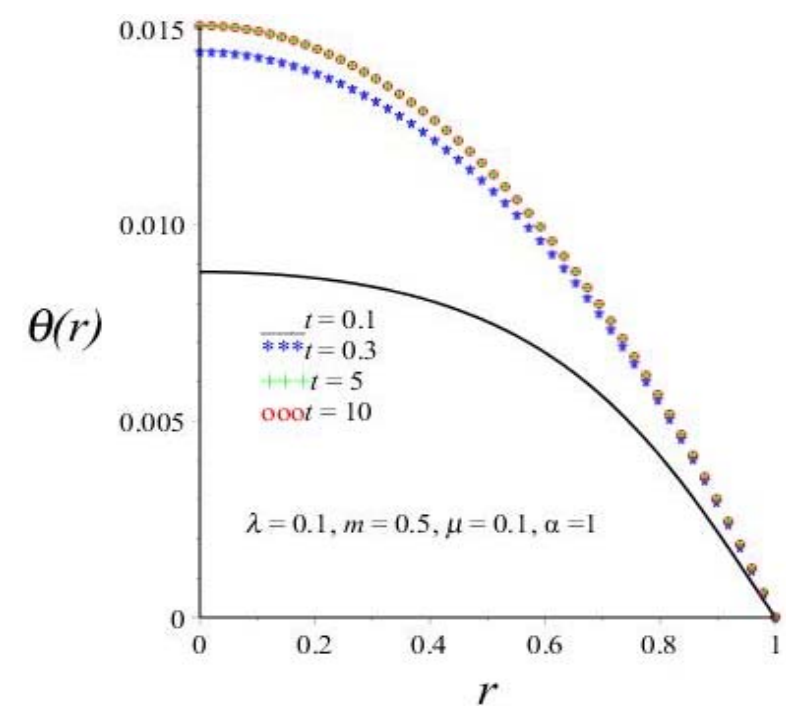

Fig. 3 Temperature variation with time. 


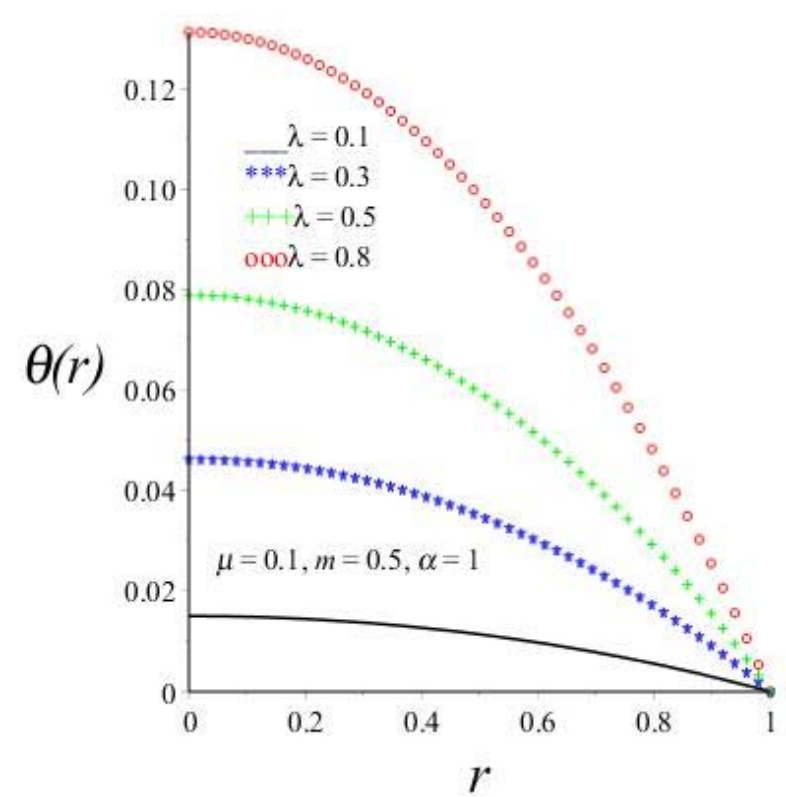

Fig. 4 Temperature variation with $\lambda$.

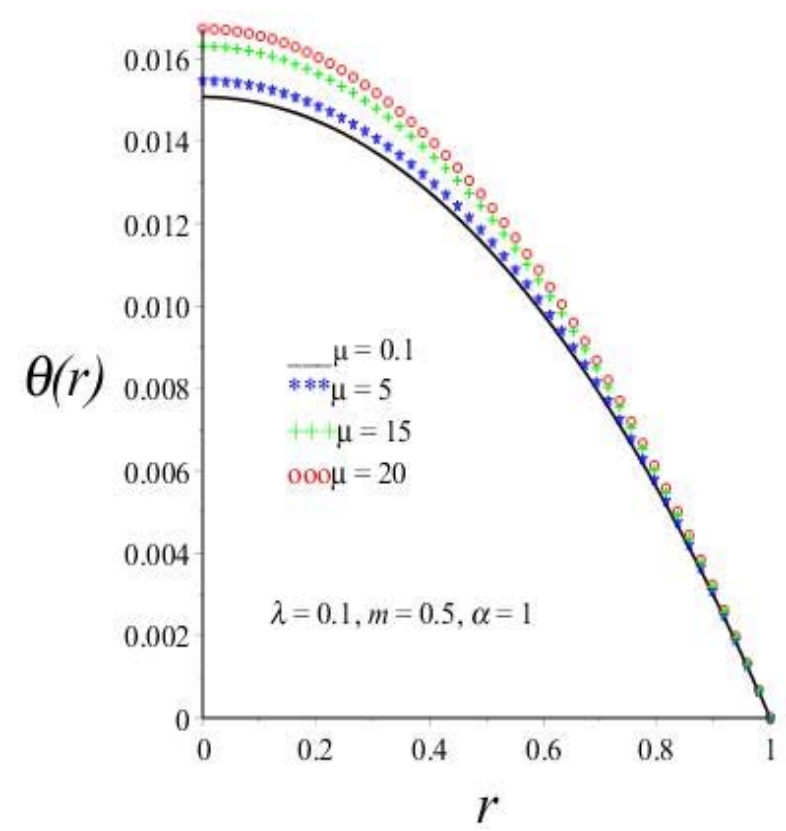

Fig. 5 Temperature variation with $\mu$.

loss of heat to the surrounding, more heat can accumulate within the system to cause self-ignited fires. We see the same result of temperature increase in Fig. 5 as the activation energy parameter $\mu$ is increased. In this case $\mu$ has no strong effect on temperature behavior as compared to $\lambda$. If $\mu$ is increased with small values, its effect on temperature behavior may not be observed, hence high values employed to show the effect. A different scenario is

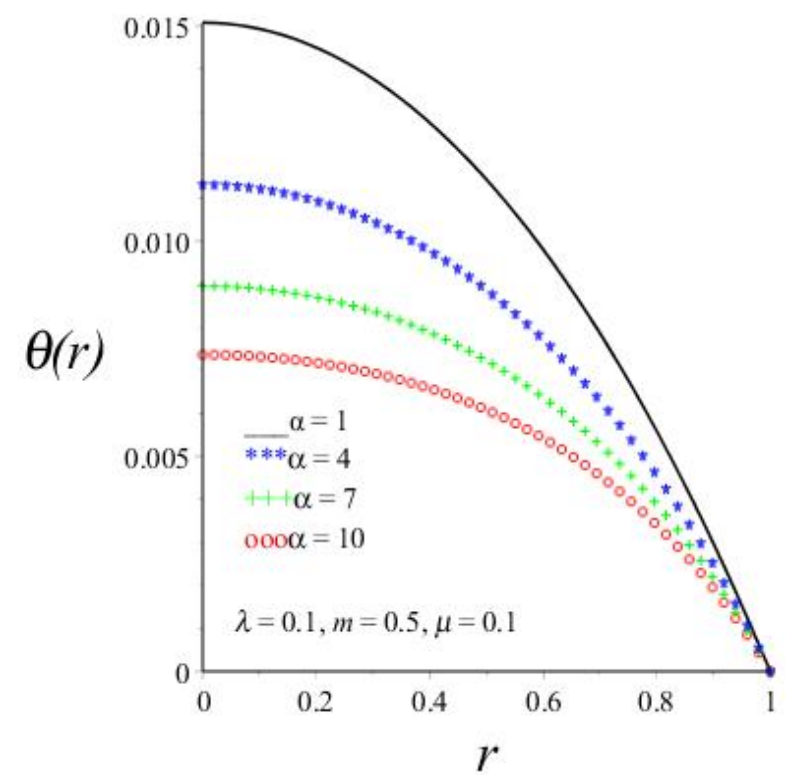

Fig. 6 Temperature variation with $\alpha$.

observed in Fig. 6, where an increase in $\alpha$, the heat loss parameter, shows a decrease in temperature fields. This parameter indicates that considerable heat loss to the ambient encourages the system's thermal stability and that the risk of self-ignited fires is minimized.

\section{Conclusion}

Analysis of transient heat in a stockpile of reactive material was investigated in this case. The study was modelled in a spherical domain. Thermo-physical parameters embedded within the system that have an effect on temperature behavior during exothermic chemical reaction in a sphere, were identified as $\lambda, \mu$ and $\alpha$ for $t>0$. It was found that an increase on parameters $\lambda$ and $\mu$ increases the temperature of the sphere during the exothermic chemical reaction whereas $\alpha$ shows an opposite effect, which is of importance to attain thermal stability of the system. In general, the thermal stability in a sphere of reactive material is far much better as compared to that in a cylindrical set up and rectangular slab, and this is enhanced by the fact that no effect of the numerical index $m$ on temperature behavior is observed in a sphere for the values $-2,0$ and 0.5 . The significance of this theoretical investigation of self-igniting processes is to provide a cheaper and simpler way of 
understanding factors that enhance temperature increases or vice-versa, by using mathematical approach. In this case, the study was simplified by considering a one dimensional partial differential equation. The study can be extended to more complicated situation using a two dimensional or a three dimensional setup.

\section{References}

[1] Lebelo, R. S. 2014. "Numerical Investigation of $\mathrm{CO}_{2}$ Emission and Thermal Stability of a Convective and Radiative Stockpile of Reactive Material in a Cylindrical Pipe of Variable Thermal Conductivity.” In AIP Conference Proceedings, 60-8.

[2] Lebelo, R. S., and Makinde, O. D. 2015. "Numerical Investigation of CO2 Emission and Thermal Stability of a Convective and Radiative Stockpile of Reactive Material in a Cylindrical Pipe." Advances in Mechanical Engineering 7 (12): 1-11.

[3] Makinde, O. D., Chinyoka, T., and Lebelo, R. S. 2011. "Numerical Investigation into $\mathrm{CO}_{2}$ Emission, $\mathrm{O}_{2}$ Depletion, and Thermal Decomposition in a Reacting Slab.” Mathematical Problems in Engineering 2011 (2011): 1-19.

[4] Lebelo, R. S. 2015. "Convective and Radiative Heat Loss Impact on $\mathrm{CO}_{2}$ Emission, $\mathrm{O}_{2}$ Depletion and Thermal Stability in a Reactive Slab of Variable Thermal Conductivity.” IEEE Conference Record 36345.

[5] Lohrer, C., Krause, U., and Steinbach, J. 2005. "Self-ignition of Combustible Bulk Materials under Various Ambient Conditions.” Special Issue on Energy from Waste 83 (2): 145-50.

[6] Lebelo, R. S., and Makinde, O. D. 2015. "Modelling the Impact of Radiative Heat Loss on $\mathrm{CO}_{2}$ Emission, $\mathrm{O}_{2}$ Depletion and Thermal Stability in a Reactive Slab.” Transactions of Mechanical Engineering 39 (2): 351-65.

[7] Dainton, F. S. 1960. Chain Reaction: An Introduction. New York: Wiley.

[8] Warnatz, J., Maas, U., and Dibble, R. 2001. Combustion: Physical and Chemical Fundamentals, Modeling and Simulation, Experiments, Pollutant Formation. Berlin: Springer and Co. K.

[9] Williams, F. A. 1985. Combustion Theory. Menlo Park: Benjamin \& Cuminy Publishing.

[10] Sadiq, M. A., and Merkin, J. H. 1994. "Combustion in a Porous Material with Reactant Consumption: The Role of the Ambient Temperature.” Math Comput Model 20: 27-46.

[11] Liao, S., Su, J., and Chwang, A. T. 2006. "Series Solutions for a Nonlinear Model of Combined Convective and Radiative Cooling of a Spherical Body.” Int J Heat Mass Tran 49: 243-45.

[12] Hamza, B. H., Massawe, E. S., and Makinde, O. D. 2011. "Analysis of Transient Heating Due to Exothermic Reaction in a Stockpile of Combustible Material." International Journal of the Physical Sciences 6 (18): 4337-41.

[13] Makinde, O. D. 2012. "Hermite-Pide Approach to Thermal Stability of Reacting Masses in a Slab with Asymmetric Convective Cooling.” Journal of Franklin Institute 349: 957-65G. Eason, B. Noble, and I. N. Sneddon, "On certain integrals of Lipschitz-Hankel type involving products of Bessel functions,” Phil. Trans. Roy. Soc. London, vol. A247, pp. 529-551, April 1955. (references). 